\title{
Presumed tuberculosis- associated uveitis: rising incidence and widening criteria for diagnosis in a non-endemic area
}

N Krassas ${ }^{1}$, J Wells' ${ }^{1}$, C Bell ${ }^{2}$, M Woodhead ${ }^{2,3}$ and $\mathrm{N}$ Jones ${ }^{1,3}$

\begin{abstract}
Purpose To assess the incidence, clinical ocular involvement and effectiveness of antituberculous treatment in patients with chronic uveitis presumed to be associated with tuberculosis in a non-endemic community. Patients and methods Retrospective case series of patients with uveitis and evidence of tuberculosis, with no other identified cause of uveitis, who underwent a 6-month course of standard anti-tuberculosis treatment between 2008 and 2015. The response to treatment was assessed at $\mathbf{6}$ and $\mathbf{1 2}$ months after initiation of treatment.

Results Forty-eight patients were included of whom $36(75 \%)$ were born outside the United Kingdom. Only five had concurrent active pulmonary or nodal tuberculosis. There were 85 affected eyes, including 25 with granulomatous anterior uveitis, 32 with retinal vasculitis (occlusive in 21), and 20 with multifocal choroiditis or serpiginous-like retinochoroiditis. Gamma-interferon testing was positive in $95 \%$. Complete resolution at end point was seen in only $60 \%$, but a further $19 \%$ were inflammationfree on topical steroid only. Resolution was lower $(50 \%)$ in those with panuveitis compared to other anatomical types (75\%). Sixty-four eyes (75\%) had a LogMAR visual acuity of 0.1 or better at the end of the study.

\section{Introduction}

Tuberculosis (TB) remains a common global disease, but in England the incidence of new cases is low and has been reducing since a peak of 8280 cases in 2011, a total of 5758 cases being notified in 2015 (the most recent year for which figures are available) ${ }_{1}^{1}$ giving an overall incidence of 10.5/100 000. Within England, the areas of highest incidence are in London, Leicester, Birmingham, and Manchester. Extrapulmonary TB (within which ocular involvement is classified by Public Health England) accounts for $58 \%$ of cases ${ }^{1}$ and has proportionately increased in recent years.

The incidence and treatment outcomes of presumed TB-associated uveitis (TBU) up to 2007 have previously been reported from this centre. ${ }^{2}$ Subsequently an increase in cases has been diagnosed, and recently described clinical manifestations are increasingly experienced. ${ }^{3}$ This study aims to document the changing incidence of TBU in Manchester, to describe the clinical signs at diagnosis, and to document outcomes using the National Institute for Clinical Excellence recommended 6-month antituberculosis treatment (ATT) regime for $\mathrm{TB}^{4}{ }^{4}$ which we routinely give to all those identified with TB-associated uveitis.
\end{abstract}

Conclusions The incidence of presumed tuberculosis-associated uveitis (TBU) has almost quadrupled in this region. The efficacy of treatment has not been enhanced by the introduction of gamma-interferon testing to support diagnosis. Some patients may require more prolonged antibiotic therapy to ensure quiescence, but chronic non-infective anterior uveitis may in any case follow treated TBU. Eye (2018) 32, 87-92; doi:10.1038/eye.2017.152; published online 4 August 2017

\section{Patients and methods}

Patients with uveitis and possible TB diagnosed between January 2008 and July 2015 were identified retrospectively from the database of the Manchester Uveitis Clinic, into which data are entered prospectively and stringently by one of us (NPJ). These were cross-referenced with the Manchester Royal Infirmary Tuberculosis Unit (which prescribes and supervises ATT for
${ }^{1}$ Manchester Royal Eye Hospital, Central Manchester University Hospitals NHS Foundation Trust, Manchester, UK

${ }^{2}$ Department of Respiratory Medicine, Manchester Royal Infirmary, Central Manchester University Hospitals NHS Foundation

Trust, Manchester, UK

${ }^{3}$ Medical Academic Health Science Centre, University of Manchester,

Manchester, UK

Correspondence:

$\mathrm{N}$ Jones, Ophthalmology, Manchester Royal Eye Hospital, Oxford Road, Manchester M13 9WL, UK Tel: +0161 276 5628; Fax: +01612726618 E-mail: nicholas.jones@ cmft.nhs.uk

Received: 8 November 2016 Accepted in revised form: 30 April 2017 Published online: 4 August 2017 
patients with TBU), but this did not identify further cases, and we are confident that case identification is complete. Patients were classified with TBU if, in the presence of qualifying uveitis, there was either evidence of active TB at another site, or a history of previous $\mathrm{TB}$, or an immune response to $\mathrm{TB}$ antigens (as a tuberculin skin test (Mantoux), or gamma-interferon test (GIFN), or both) if no other cause of uveitis was identified. Qualifying uveitis included any uveitis with granulomatous keratic precipitates or iris nodules, any steroid-resistant uveitis, intermediate uveitis, retinal vasculitis with or without occlusion, multifocal choroiditis, single choroidal tubercle, uveitis with papillitis, atypical placoid, or serpiginous-like retinochoroiditis. Patients were included in the study only if a full 6-month course of standard ATT was administered (2 months of rifampicin, isoniazid, pyrazinamide, and ethambutol followed by 4 months of rifampicin and isoniazid). ${ }^{4}$ Treatment compliance was ensured by home visits, and patients attended the TB clinic monthly.

Data were extracted from clinical records including age, sex, ethnic origin, country of birth, history of previous TB or contact, Mantoux testing, and/GIFN (QuantiFERONTB Gold In-Tube is used in this Trust) ACE levels and treatment details. Ocular status including visual acuity (VA) and type of inflammation were assessed at the commencement of ATT (baseline), at the completion of treatment (6 months), and 6 months later (12 months). Details of concurrent oral steroid treatment and/or immunosuppression were noted.

\section{Results}

Tuberculosis-associated uveitis was considered in 91 patients over the study period. From these, the following were excluded: 12 patients demonstrated evidence of latent TB but the uveitis was considered unassociated, and either no ATT, or 3 months of prophylaxis for latent TB were given; in nine patients there was evidence of latent TB and previous multifocal choroiditis or atypical placoid retinochoroiditis but no current activity (in five of whom ATT had previously been given) and again, ATT was not necessary for uveitis; in three cases, the diagnosis of sarcoidosis was made, following previous treatment for $\mathrm{TB}$, and in two cases, clinical information was inadequate. Overall, 65 were diagnosed with presumed TBU and received ATT. Of these, two did not complete a full 6-month ATT course, two received extended ATT following previous treatment failure, seven defaulted from follow-up before 12-month data were recorded and the care of five was transferred elsewhere so that data was incomplete. These 17 were also excluded from full data analysis, but interim (6-month) outcomes for seven of these are included. The remaining 48 patients were presumed to have TBU, received a full 6-month course of
ATT and were followed for 12 months. Of these, 23 (48\%) were male and 25 female, with a mean age of 34.5 years at presentation (range, 14-60 years).

All 48 patients were UK resident; 12 were UK born (of whom only 3 were of white British lineage), 10 were born in India, 12 in Pakistan (total 46\% born in India or Pakistan), 11 (23\%) in Africa, 2 in the Philippines, and 1 in Saudi Arabia. Thirty-three (69\%) were of Indo-Pakistani or Asian ethnicity, 3 were Caucasian and 12 (25\%) were Afro-Caribbean. Intraocular inflammation was bilateral in 37 patients (73\%) and there were in total 85 affected eyes. At presentation, eight patients had anterior uveitis only (6 granulomatous, 2 non-granulomatous steroidresistant), 4 had intermediate uveitis, 24 (50\%) panuveitis, 12 posterior uveitis. A total of 38 patients had anterior segment inflammation. Of these, 15 (31\%) exhibited granulomatous inflammation. In the posterior segment, 20 patients (32 eyes) had retinal vasculitis (occlusive in 21 eyes, with disc or retinal neovascularisation in 10 eyes; 12 patients required Argon laser photocoagulation), 11 eyes of 8 patients had multifocal choroiditis, 9 eyes of 5 patients had atypical placoid or serpiginous-like chorioretinitis, and 7 eyes of 5 patients had papillitis.

Previous BCG vaccination had been received by 25 patients (52\%). Two patients had previously been treated for TB (one pulmonary, one nodal). Eleven (23\%) had known contact with a family member treated for TB, but only one had received TB prophylaxis. Three others were nurses who had worked with TB patients. In 40 patients, the chest X-ray was normal; in the remaining eight, six showed signs indicative of probable old $\mathrm{TB}$, one showed features consistent with active upper-lobe pulmonary TB, and one showed presumed TB-related pleural effusion.

Mantoux testing was performed in 34 patients; of these, 14 had a $20 \mathrm{~mm}$ or greater induration diameter, 15 had $15-19 \mathrm{~mm}$, the remainder showing $14,14,12,11$, and $8 \mathrm{~mm}$. All of the latter five patients had positive GIFN tests. Of those, 14 patients not undergoing Mantoux testing, two had prior TB. The remainder did not undergo Mantoux testing for organisational reasons (usually because patients were unwilling to return for result reading), and all had positive GIFN tests. Gammainterferon testing was performed in 45 patients (of the three not tested, one had previously treated $\mathrm{TB}$, one was HIV positive, and one was Mantoux-tested in another hospital) and was positive in 43 (95\%). Two patients showed negative GIFN; one had active pulmonary TB and a Mantoux of $20 \mathrm{~mm}$; the other had bilateral granulomatous panuveitis and a Mantoux of $17 \mathrm{~mm}$. Overall, five patients $(10 \%)$ were considered to have concurrent active pulmonary or nodal TB. Thirty-three patients underwent both Mantoux and GIFN testing. Using the UK Mantoux interpretation guidelines, ${ }^{4}$ the concordance between tests was 30/33 (91\%). 
The angiotensin-converting enzyme (ACE) level was measured at presentation in 44 patients with a mean of $56.3 \mathrm{IU} / 1$. Of these, 19 (43\%) showed an elevated level $>55 \mathrm{IU} / 1$ with all except one of these in the range 56-90. These results are shown in Figure 1, compared to the ACE levels found in 112 patients from this clinic diagnosed with sarcoidosis. ${ }^{5}$

All 48 patients completed a full 6-month weightadjusted course of ATT (2 months of rifampicin, isoniazid, pyrizinamide, and ethambutol (Voractiv or Rifater with ethambutol) followed by 4 months of rifampicin and isoniazid (Rifinah)). Topical steroids were used as required in 36 patients with anterior segment involvement. Oral prednisolone was used in 21 patients (44\%); of these, 3 had used oral steroids for several months prior to the diagnosis of TBU and commencement of ATT; 6 commenced prednisolone $30-80 \mathrm{mg} /$ day at the same time as ATT; 11 commenced prednisolone 30-80 mg/day 1-4 weeks after commencing ATT, and

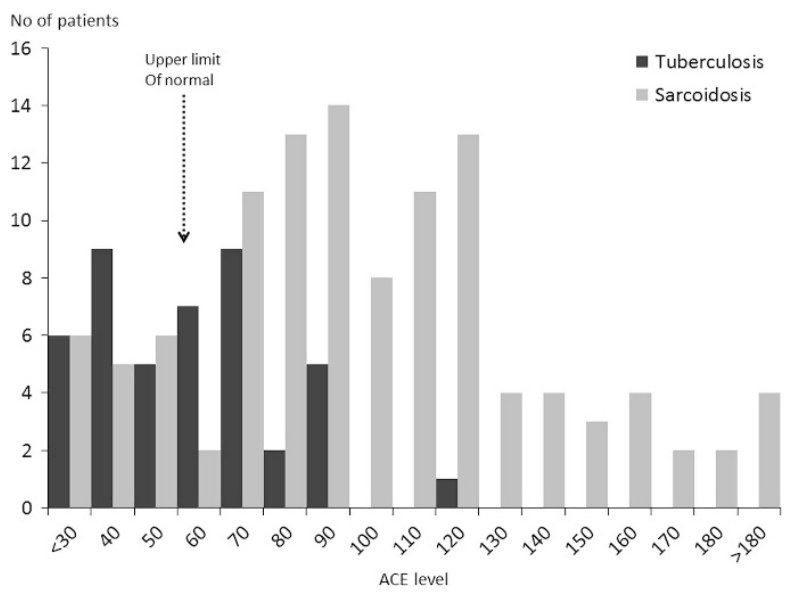

Figure 1 ACE levels in 44 patients with presumed ocular tuberculosis, compared to 112 patients with sarcoidosis diagnosed in this clinic.
1 commenced prednisolone $100 \mathrm{mg}$ /day 4 months after starting ATT, following inflammation recurrence. Six months after starting ATT, 10 patients were still using prednisolone, and 6 months later, 3 remained on oral steroid. All patients were using rifampicin for 6 months and because of liver enzyme induction, prescribed steroid doses were doubled during that period. Two patients were commenced on azathioprine immunosuppression after discontinuing ATT; one patient was immunosuppressed with mycophenolate and ciclosporin during ATT, and this was discontinued before a 12-month assessment.

During the 12-month observation period, 4 patients with occlusive retinal vasculitis developed vitreous haemorrhage, 12 underwent argon laser retinal ablation, and 1 required pars plana vitrectomy with pre-retinal membrane delamination. Ten developed macular oedema. Eleven patients developed secondary glaucoma, five glaucoma drainage implants were inserted, and one underwent cyclodiode treatment. Seven patients developed cataract and nine cataract procedures were performed. The clinical manifestations and complications of TBU in all eyes are summarised in Table 1.

After a completed course of 6 months ATT, 37/55 patients $(67 \%)$ had no intraocular inflammation (with or without topical and/or systemic steroid treatment). Six months later (12 months after commencing ATT), 29/48 patients $(60 \%)$ had no intraocular inflammation, and had discontinued all treatment. A further nine patients had no active intraocular inflammation, and were using topical steroid only. Nine patients were using topical steroid and had mild active anterior uveitis only, and three were using oral steroid (of which two were also using oral immunosuppression). At the end of the study, 38 patients (79\%) had no active intraocular inflammation. The rate of inflammation recovery was separately analysed for anatomical type of uveitis (anterior, intermediate,

Table 1 Clinical manifestations and complications of tuberculosis-associated uveitis in 85 eyes

\begin{tabular}{lcl}
\hline Uveitis/complication type & No of eyes $(\%)$ & Comments \\
\hline Anterior uveitis only & $13(15.3)$ & $8 / 13$ granulomatous AU \\
Intermediate uveitis & $8(9.4)$ & $0 / 8$ granulomatous AU \\
Posterior uveitis & $20(23.5)$ & 12 retinal vasculitis, 5 multifocal choroiditis \\
Panuveitis & $44(51.7)$ & $13 / 40$ granulomatous AU \\
Serpiginous-like choroiditis & $9(10.6)$ & 7 panuveitis, 2 posterior uveitis \\
Papillitis & $7(8.2)$ & 2 AU, 3 posterior uveitis, 2 panuveitis \\
Multifocal choroiditis & $11(12.9)$ & 7 panuveitis, 5 posterior uveitis \\
Retinal vasculitis & $32(37.6)$ & $15 / 32(47 \%)$ occlusive \\
(occlusive) & $21(24.7)$ & 2 NVD, 14 NVE, 17 laser, 4 Vit haem, 1 vitrectomy \\
Secondary glaucoma & $13(15.7)$ & 9 panuveitis, 2 AU, 1 intermediate uveitis \\
Secondary cataract & $11(13.2)$ & 5 underwent surgery (4 Baerveldt GDI, 1 Ahmed GDI) \\
\hline
\end{tabular}

Abbreviations: AU, anterior uveitis; GDI, glaucoma drainage implant; NVD, new vessels elsewhere; NVD, new vessels at the disc; Vit haem, vitreous haemorrhage. 


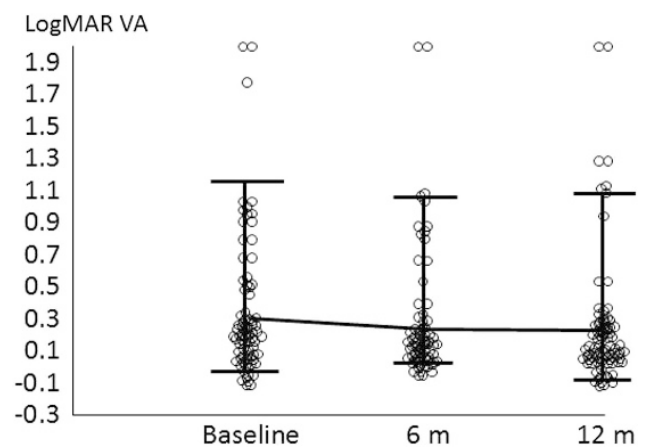

Figure 2 Change in LogMAR visual acuity for 85 affected eyes from baseline (commencement of anti-tuberculosis treatment) and at 6 and 12 months.

posterior, and panuveitis) and for clinical characteristics (granulomatous anterior uveitis, retinal vasculitis (occlusive and non-occlusive), serpiginous-like retinochoroiditis, multifocal choroiditis, and papillitis). Complete resolution (no inflammation, and off all treatment) was seen in $75 \%$ of all patients with anterior, intermediate, or posterior uveitis, but in only $50 \%$ of those with panuveitis. For individual characteristics, the highest inflammation resolution rate $(67 \%)$ was seen for those with non-occlusive retinal vasculitis, and the lowest (20\%) for those with serpiginous-like retinochoroiditis. However, these samples were small and no differential resolution rates reached statistical significance.

The change in LogMAR VA for the 85 affected eyes is shown in Figure 2. At 12 months after commencing ATT, mean VA improved from 0.30 to 0.22 , which was not significant $(P=0.95)$. Thirty-nine eyes $(45.9 \%)$ improved by 0.1 LogMAR and 21 eyes $(24.7 \%)$ by 0.2 LogMAR. Sixty-four eyes (75.3\%) ended with a LogMAR acuity of 0.1 or better. The causes of severe central visual loss (LogMAR 1.0 or worse) included unoperated vitreous haemorrhage (one eye), unoperated cataract (one eye), glaucomatous visual field loss (one eye), and macular scarring (five eyes), a total of eight eyes.

Forty-eight patients are reported here, but including those others with new, active TBU in whom data were inadequate for full analysis, a total of 65 patients presented over January 2008-June 2015 (8.6 new patients per year). This compares with 40 over 17 years (19922007) in a previous study from this centre ${ }^{2}$ (2.3 per year). The incidence of all new tuberculosis cases, and of TBU in Manchester since 2003 (the earliest year for which full incidence figures are available), are shown in Figure 3.

\section{Discussion}

The incidence of new cases of TBU seen here over a 7.5-year period, compared to a previous study ${ }^{2}$ has

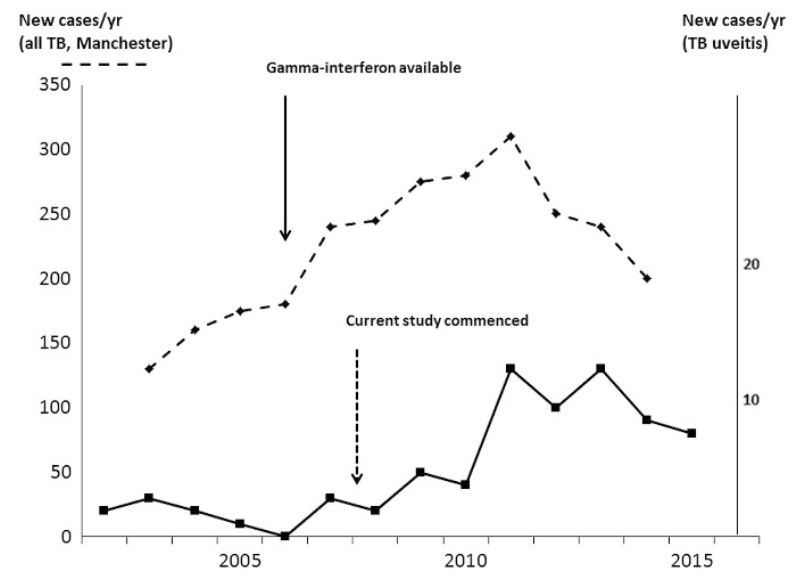

Figure 3 The annual incidence of all TB (dotted line) and presumed ocular tuberculosis (solid line) presenting in Manchester 2002-2015.

almost quadrupled. During this period, the overall incidence of tuberculosis in England peaked in 2011 since when there has been a year-on-year fall. However, it is likely that TBU diagnostic rates are rising because of increasing recognition of the possibility of TBU, especially for serpiginous-like and atypical placoid retinochoroiditis. 3,6 Anatomically, panuveitis was the most common distribution of uveitis in this series. Gamma-interferon testing was used during the whole period of this study (GIFN is now recommended for supplementary testing in those who are Mantouxpositive $\left.{ }^{4}\right)$; diagnosis has been assisted by this testing method because confounding by previous BCG vaccinated can be eliminated. There may be significant discordance between GIFN and Mantoux testing ${ }^{7}$ but the tests are complementary, and this is probably an argument for retaining both in diagnosis. ${ }^{8}$ In this case series, concordance was 91\% using Quantiferon Gold, which appears to be more specific than T-spot testing. ${ }^{9}$

There are no internationally agreed diagnostic criteria for $\mathrm{TBU}^{10}$ and this is reflected in varying inclusion criteria between studies. Attempts have been made to stratify clinical signs suggestive of diagnosis ${ }^{11}$ but a demonstration of differential cure rates for different clinical manifestations, is necessary. The sample size in this case series has been inadequate for this purpose, and a multicentre Collaborative Ocular Tuberculosis Study is in the planning stage. However, there is no indication that any of the signs used here is diagnostically inferior for TBU. Our finding that eyes with panuveitis are less likely to respond to treatment has been reported elsewhere. ${ }^{12}$

There has been a change in the clinical manifestations of TBU seen in this clinic. A previous case series up to $2007^{2}$ contained 1/27 (4\%) with serpiginous-like choroiditis compared to $5 / 42(12 \%)$ in this series. This new 
manifestation of TBU was first observed in India in 1997 and first described in 2003, ${ }^{6}$ being confirmed and clinically defined by others. ${ }^{13}$ We first observed a case in 2003 and have since described the rising incidence in Manchester. ${ }^{3}$ The phenomenon is now widely recognised as an important indicator of TBU and should provoke diagnostic testing.

Elevated ACE levels are well-reported in patients with TB. However, it is also recognised, for those with pulmonary disease, that levels tend not to be elevated to the extent often seen in sarcoidosis. A previous study ${ }^{14}$ showed mean ACE levels of 58.6 and 104.4 IU/1 for TB and sarcoidosis, respectively. This study (mean ACE levels $56.3 \mathrm{IU} / 1$ in TB and $94.3 \mathrm{IU} / 1$ in sarcoidosis, Figure 1) suggests that ACE elevation demonstrates a similar relationship for those with TB and sarcoidosis presenting with intraocular inflammation. This distinction however does not permit an ACE level to be used as a diagnostic discriminator between sarcoidosis and TB for an individual presenting with uveitis.

In this study, disappointingly only $60 \%$ of patients were free of inflammation, and off all treatment, 6 months after completing ATT. However, in the great majority of patients with ongoing inflammation, only non-granulomatous anterior uveitis remained and only three patients required oral treatment. Overall, 38 patients $(79 \%)$ had no active intraocular inflammation detectable at the final assessment. Arguably these are good outcomes, but the need to define mild ongoing anterior uveitis as 'treatment failure' highlights the need for accepted criteria for 'success' in TBU treatment. All patients in this case series received identical (weightadjusted) ATT for 6 months. Elsewhere, variable treatment has been used, including extended treatment for 9 or 12 months. ${ }^{15}$ Concern has been expressed about the potential increased risk of ethambutol in those with uveitis, and moxifloxacin has been substituted. ${ }^{16}$ Our view is that this risk is small, poorly defined, and does not justify treatment change for this reason alone. While there seems little doubt that ATT enhances outcomes in TBU patients in comparison to oral steroid alone, ${ }^{17}$ the optimal treatment duration remains uncertain.

There are several possible reasons for residual or recurrent uveitis after ATT for presumed TBU. ${ }^{18}$ First, TBU was the correct diagnosis but either 6 months' treatment is inadequate or organisms are drug-resistant, so that viable organisms remain and a longer course or different drug combination should be considered. This suggestion has been previously made ${ }^{15,18}$ and there is no international consensus on management. ${ }^{10}$ The second possible explanation for post-treatment uveitis is the phenomenon of presumed chronic autoimmune anterior uveitis following (and presumably triggered by) previous intraocular infection. This has been reported after necrotising viral retinitis ${ }^{19}$ but is also wellrecognised following other intraocular infections including toxoplasmosis. This may explain at least some of those with residual non-granulomatous uveitis confined to the anterior segment (and therefore would not indicate inadequately effective antibacterial treatment). A third, very unlikely possibility is that an as-yet unidentified mycobacterium which provokes a GIFN response, but which is not adequately responsive to standard ATT, could cause some cases of intraocular inflammation. This is currently only hypothetical, but M. tuberculosis is not the only pathogen to cause TB (which may also arise, very uncommonly, from M. bovis). The fourth possibility is that the intraocular infection is not caused by any mycobacterium, but has a different, unknown cause. A positive GIFN test would therefore indicate either latent TB, with prior infection unrelated to the current uveitis, or a false positive GIFN test (which may be caused by, amongst others, some commensal mycobacteria, HIV infection or defective antigen tubes. ${ }^{20}$

A previous case series from this $\mathrm{clinic}^{2}$ found that $70 \%$ of patients were inflammation-free 6 months after completing ATT. Gamma-interferon testing became available only late during that study period and only $15 \%$ patients underwent testing. In comparison, $94 \%$ of patients in this case series underwent GIFN testing, providing near-confirmation of prior infection and reducing any confounding caused by prior BCG vaccination and Mantoux interpretation. ${ }^{21}$ It is therefore disappointing that only $58 \%$ resolved completely after $\mathrm{ATT}$, and this suggests either that accurate diagnosis is still not being achieved, or that 6 months of ATT is an inadequate duration, or that some cases of TBU are caused by drug-resistant TB, or that intraocular inflammation may persist despite resolution of TB. It has been suggested that higher GIFN responses are associated with higher recovery rates, ${ }^{22}$ indicating a possible quantitative effect from GIFN testing.

Unfortunately, PCR testing of intraocular fluid is not permitted at this hospital, but it is possible that PCR testing of either devR or MPB64 would significantly improve detection rates, both of these showing 100\% specificity in a small series. ${ }^{23}$

In conclusion, presumed tuberculosis-associated uveitis is of increasing importance in the United Kingdom and current diagnosis and management remain unsatisfactory. Currently, Public Health England (PHE) does not report ocular TB as a separate entity. There is however increasing recognition of the importance of ocular involvement and some TB authorities, including Manchester, ${ }^{24}$ do report TBU, and in this city, TBU is, 
after nodal TB, the commonest recorded form of extrapulmonary TB. Several parties are working to ensure that ocular TB is recorded routinely on PHE annual statistics.

\section{Summary}

\section{What was known before}

- Tuberculosis can cause uveitis and the disease is probably under-diagnosed. Preferred best management is not agreed.

\section{What this study adds}

- Tuberculous uveitis has substantially increased in incidence in the UK. Gamma interferon testing can confirm exposure in patients with uveitis without concurrent active disease. Currently accepted 6-month treatment course may be inadequate.

\section{Conflict of interest}

The authors declare no conflict of interest.

\section{Acknowledgements}

This research was facilitated by the Greater Manchester Local Clinical Research Network.

\section{References}

1 Davidson JA, Lalor MK, Mohiyuddin T, Loutet MG, Uddin T, Venugopalan S et al. Tuberculosis in England 2016 Report. Public Health England: London, 2016.

2 Sanghvi C, Jones NP, Bell C, Woodhead M, Hardy C. Presumed tuberculous uveitis: diagnosis, management and outcome. Eye 2011; 25: 475-480.

3 Gan W-L, Jones NP. Serpiginous-like choroiditis as a marker for tuberculosis in a non-endemic area. Br J Ophthalmol 2013; 97: 644-647.

4 Anon. Tuberculosis. NICE Guideline NG33, 2016. National Institute for Clinical Excellence ISBN 978-1-4731-1587-3.

5 Jones NP, Tsierkezou L, Patton N. Lymphopenia as an independent predictor of sarcoidosis in patients presenting with uveitis. Br J Ophthalmol 2016; 100: 1393-1396.

6 Gupta V, Gupta A, Arora S, Bambery P, Dogra MR, Agarwal A. Presumed tubercular serpiginouslike choroiditis: clinical presentations and management. Ophthalmology 2003, 110: 1744-1749.

7 Ang M, Kiew SY, Wong WL, Chee SP. Discordance of two interferon- $\gamma$ release assays and tuberculin skin test in patients with uveitis. Br J Ophthalmol 2014; 98: 1649-1653.

8 Llorenç V, González-Martin J, Keller J, Rey A, Pelegrín L, Mesquida $\mathrm{M}$ et al. Indirect supportive evidence for diagnosis of tuberculosis-related uveitis: from the tuberculin skin test to the new interferon gamma release assays. Acta Ophthalmol 2013; 91: e99-e107.

9 Ang M, Wong WL, Kiew SY, Li X, Chee SP. Prospective head-to-head study comparing 2 commercial interferon gamma release assays for the diagnosis of tuberculous uveitis. Am J Ophthalmol 2014; 157: 1306-1314.

10 Lou SM, Larkin KL, Winthrop K, Rosenbaum JT. Uveitis Specialists Study Group. Lack of consensus in the diagnosis and treatment for ocular tuberculosis among uveitis specialists. Ocul Immunol Inflamm 2015; 23: 25-31.

11 Ang M, Hedayatfar A, Zhang R, Chee SP. Clinical signs of uveitis associated with latent tuberculosis. Clin Exp Ophthalmol 2012; 40: 689-696.

12 Agrawal R, Gupta B, Gonzalez-Lopez JJ, Rahman F, Phatak S, Triantafyllopoulou I et al. The role of antitubercular therapy in patients with presumed ocular tuberculosis. Ocul Immunol Inflamm 2015; 23: 40-46.

13 Vasconcelos-Santos DV, Rao PK, Davies JB, Sohn EH, Rao NA. Clinical features of tuberculous serpiginouslike choroiditis in contrast to classic serpiginous choroiditis. Arch Ophthalmol 2010; 128: 853-858.

14 Khan AH, Ghani F, Khan A, Khan MA, Khurshid M. Role of serum angiotensin converting enzyme in sarcoidosis. J Pak Med Assoc 1998; 48: 131-133.

15 Ang M, Hedayatfar A, Wong W, Chee SP. Duration of antitubercular therapy in uveitis associated with latent tuberculosis: a case-control study. Br J Ophthalmol 2012; 96: 332-336.

16 Potter J, Agrawal R, Barraclough C, Rahman F, Westcott M, Pavesio CE et al. Moxifloxacin: an alternative to ethambutol for the treatment of presumed ocular tuberculosis. Ocul Immunol Inflamm 2016; 24: 508-514.

17 Bansal R, Gupta A, Gupta V, Dogra MR, Bambery P, Arora SK. Role of anti-tubercular therapy in uveitis with latent/manifest tuberculosis. Am J Ophthalmol 2008; 146: 772-779.

18 Basu S, Nayak S, Padhi TR, Das T. Progressive ocular inflammation following anti-tubercular therapy for presumed ocular tuberculosis in a high-endemic setting. Eye 2013; 27: 657-662.

19 Comer M, Young S, Lightman S. Anterior uveitis after healed acute retinal necrosis. Arch Ophthalmol 2002; 120: 88-90.

20 Slater M, Parsonnet J, Banaei N. Investigation of falsepositive results given by the QuantiFERON-TB Gold In-Tube assay. J Clin Microbiol 2012; 50: 3105-3107.

21 Mahan CS, Johnson DF, Curley C, van der Kuyp F. Concordance of a positive tuberculin skin test and an interferon gamma release assay in bacille Calmette-Guérin vaccinated persons. Int J Tuberc Lung Dis 2011; 15: 174-178.

22 Agrawal R, Gonzalez-Lopez JJ, Nobre-Cardoso J, Gupta B, Grant R, Addison PK et al. Predictive factors for treatment failure in patients with presumed ocular tuberculosis in an area of low endemic prevalence. Br J Ophthalmol 2016; 100: 348-355.

23 Kataria P, Kumar A, Bansal R, Sharma A, Gupta V, Gupta A. devR PCR for the diagnosis of intraocular tuberculosis. Ocul Immunol Inflamm 2015; 23: 47-52.

24 Bell C. Tuberculosis in Manchester and Trafford Annual Report 2014. Dept Resp Med, Central Manchester Foundation Trust, 2015. 